JOURNAL OF THE

AMERICAN MATHEMATICAL SOCIETY

Volume 9, Number 3, July 1996

\title{
MODULAR FORMS AND DONALDSON INVARIANTS FOR 4-MANIFOLDS WITH $b_{+}=1$
}

\author{
LOTHAR GÖTTSCHE
}

\section{INTRODUCTION}

The Donaldson invariants of a smooth simply connected 4-manifold $X$ depend by definition on the choice of a Riemannian metric $g$. In case $b_{+}(X)>1$ they turn out to be independent of the metric as long as it is generic, and thus give $C^{\infty}$-invariants of $X$.

We study the case $b_{+}(X)=1$, where the invariants have been introduced in [Ko]. We denote by $\Phi_{c_{1}, N}^{X, g}$ the Donaldson invariant of $X$ with respect to a lift $c_{1} \in H^{2}(X, \mathbb{Z})$ of $w_{2}(P)$ for an $S O(3)$ bundle $P$ on $X$ with $-p_{1}(P)-3=N$. Kotschick and Morgan showed in $[\mathrm{K}-\mathrm{M}]$ that the invariants only depend on the chamber of the period point of $g$ in the positive cone $H^{2}(X, \mathbb{R})^{+}$in $H^{2}(X, \mathbb{R})$. For two metrics $g_{1}, g_{2}$ which do not lie on a wall they express $\Phi_{c_{1}, N}^{X, g_{1}}-\Phi_{c_{1}, N}^{X, g_{2}}$ as the sum over certain wall-crossing terms $\delta_{\xi, N}^{X}$, where $\xi$ runs over all classes in $H^{2}(X, \mathbb{Z})$ which define a wall between $g_{1}$ and $g_{2}$. They also make the following conjecture.

Conjecture $1.1([\mathrm{~K}-\mathrm{M}]) . \delta_{\xi, N}^{X}$ is a polynomial in the multiplication by $\xi$ and the quadratic form $Q_{X}$ on $H_{2}(X, \mathbb{Z})$ whose coefficients depend only on $\xi^{2}, N$ and the homotopy type of $X$.

John Morgan and Peter Ozsváth have told me that they are now able to prove the conjecture [M-O].

In previous joint papers [E-G1],[E-G2] with Geir Ellingsrud we have studied the wall-crossing terms $\delta_{\xi, N}^{S}$ in the case of algebraic surfaces $S$ with $p_{g}=0$. In [E-G1] we expressed (for so called good walls) the $\delta_{\xi, N}^{S}$ in terms of Chern classes of some "standard" bundles on Hilbert schemes of points on $S$, and proceeded to compute the leading 6 terms of $\delta_{\xi, N}^{S}$ (similar results were also obtained in $\left.[\mathrm{F}-\mathrm{Q}]\right)$. In $[\mathrm{H}-\mathrm{P}]$ a Feynman path integral approach to this problem is developed, and some of the leading terms of the wall-crossing formulas are determined.

In [E-G2], which builds on [E-G1], we restrict to the case of rational surfaces and use the Bott residue formula to compute the $\delta_{\xi, N}^{S}$ explicitly (with the help of a computer). As an application, using also the blowup formulas, we computed e.g. the Donaldson invariants of $\mathbb{P}_{2}$ of degree smaller than 50 .

In $[\mathrm{K}-\mathrm{L}]$ the wall-crossing formulas had already been used in combination with the blowup formulas to compute Donaldson invariants of $\mathbb{P}_{2}$ and $\mathbb{P}_{1} \times \mathbb{P}_{1}$ and to show in particular that neither $\mathbb{P}_{2}$ nor $\mathbb{P}_{1} \times \mathbb{P}_{1}$ is of simple type. Their calculations

Received by the editors August 4, 1995.

1991 Mathematics Subject Classification. Primary 57R55 ; Secondary 11F11, 14D20.

Key words and phrases. Blowup formulas, Donaldson invariants, modular forms. 
also showed that the blowup formulas impose restrictions on the wall-crossing formulas, although this is not pursued systematically there. The authors did however expect that this can be used to determine many (and possibly all) the wall-crossing formulas for rational surfaces.

In the current paper we want to show that in fact, assuming Conjecture 1.1, one can determine the $\delta_{\xi, N}^{X}$ completely for all $X$ and all walls in $H^{2}(X, \mathbb{R})^{+}$by use of the blowup formulas. We will determine a universal generating function $\Lambda(L, Q, x, t, \tau)$ which expresses all $\delta_{\xi, N}^{X}$ for all $X, N$ and $\xi$. Here $\tau$ is a parameter from the complex upper half plane, $L, Q$ and $x$ stand for the multiplication by $\xi$, the quadratic form and the class of a point, and the exponent of $t$ is the signature of $X$. It turns out that $\Lambda(L, Q, x, t, \tau)$ is an exponential expression in certain modular forms (with respect to $\tau$ ). As an application of our results we also get modular form expressions for all the Donaldson invariants of the projective plane $\mathbb{P}_{2}$. Already in $[\mathrm{K}-\mathrm{L}]$ it had been shown that the Donaldson invariants of $\mathbb{P}_{2}$ and $\mathbb{P}_{1} \times \mathbb{P}_{1}$ are determined by the wall-crossing formulas on the blowup of $\mathbb{P}_{2}$ in two points. We use instead a simple fact due to Qin: on a rational ruled surface the Donaldson invariants with respect to a first Chern class $c_{1}$ with odd restriction to a fibre vanish for a special chamber $\mathcal{C}_{F}$.

The results of this paper should be seen in comparison with the new developments of Seiberg-Witten theory [S-W],[W1] which suggest a connection between the Donaldson invariants and modular forms: the Donaldson invariants (and also the Seiberg-Witten invariants) are seen as degenerations of supersymmetric theories, parametrized by the " $u$-plane" (i.e. the modular curve $\mathbb{H} / \Gamma(2)$ ). In fact Witten informed me that he is currently trying to determine wall-crossing formulas and the Donaldson invariants of the projective plane by integrating over the $u$-plane (see also [W2]). The results should also be related to the current work [P-T] towards proving the conjectural relationship between Seiberg-Witten and Donaldson invariants.

The main tool for getting our result are the blowup formulas, which for 4manifolds with $b_{+}=1 \mathrm{I}$ learned from [K-L]. Let $\widehat{X}:=X \# \overline{\mathbb{P}}_{2}$ (e.g. if $X$ is an algebraic surface, we can take $\widehat{X}$ to be the blowup of $X$ at a point). The idea is very simple: If $\mathcal{C}$ is a chamber in $H^{2}(X, \mathbb{R})^{+}$and $\widehat{\mathcal{C}}$ is a related chamber (see below), then there is a formula relating the Donaldson invariants of $X$ with respect to $\mathcal{C}$ and those of $\widehat{X}$ with respect to $\widehat{\mathcal{C}}$. So let now $\mathcal{C}_{-}$and $\mathcal{C}_{+}$be two chambers separated by the wall $W^{\xi}$, then in general there are several walls between the related chambers $\widehat{\mathcal{C}}_{-}$and $\widehat{\mathcal{C}}_{+}$on $\widehat{X}$, but it is very easy to determine them. We can therefore express the wall-crossing term $\delta_{\xi, N}^{X}$ as follows. We apply the blowup formulas to the related chambers $\mathcal{C}_{-}, \widehat{\mathcal{C}}_{-}$and $\mathcal{C}_{+}, \widehat{\mathcal{C}}_{+}$and add up the wall-crossing terms for all walls between $\widehat{\mathcal{C}}_{-}$and $\widehat{\mathcal{C}}_{+}$. This gives recursive relations. After encoding our information into a generating function $\Lambda_{X}(L, Q, x, t, \tau)$, these recursive relations translate into differential equations, which enable us to determine $\Lambda_{X}$ up to multiplication by a universal function $\lambda(\tau)$. Unlike the case of the blowup formulas in [F-S], the modular forms enter the formulas already as the coefficients of the differential equations; they arize as theta functions for lattices describing the walls between related chambers.

In order to finally determine $\lambda(\tau)$ we consider the particular case $X=\mathbb{P}_{1} \times \mathbb{P}_{1}$. The above mentioned result of Qin now says that, for the first Chern class $c_{1}=$ $F+G$, the sum of the classes of the fibres in the two different directions, there 
are always two different chambers $\mathcal{C}_{F}, \mathcal{C}_{G}$ of type $\left(c_{1}, N\right)$ where the corresponding Donaldson invariants vanish. Therefore the sum of the $\delta_{\xi, N}^{X}$ for all classes $\xi$ defining walls between $\mathcal{C}_{F}$ and $\mathcal{C}_{G}$ must be zero. This fact gives us an additional recursion relation, and with this we can finally determine $\lambda(\tau)$.

If we assume only a weaker form of the conjecture, namely if we allow $\delta_{\xi, N}^{X}$ to depend on $X$, rather than just on the homotopy type, then we still get our result for $X$ a rational surface. If we assume the conjecture and the blowup formulas also in the case that $X$ is not simply connected but $b_{1}(X)=0$, then we can partially extend our result also to this case.

This paper was written at the Max-Planck-Institut für Mathematik and benefitted very much from the possibility to discuss with several of the experts in the field. I am very thankful to Don Zagier, who proved Lemma 4.11 for me. I would also like to thank John Morgan and Stefan Bauer for very useful conversations. I would like to thank Dieter Kotschick for sending me the preprint of [K-L], which was very important both for [E-G2] and for this work. This paper grew out of the joint work [E-G1], [E-G2] with Geir Ellingsrud. Motivated by this work, and based also on $[\mathrm{K}-\mathrm{L}]$, I slowly realized the importace of the blowup formulas in this context. Also the explicit formulas for the wall-crossing in [E-G2] were very important for me to keep confidence in my computations.

\section{BACKGROUND MATERIAL}

In this paper we will denote by $X$ a simply connected smooth 4-manifold with $b_{+}(X)=1$ and $b_{2}(X) \geq 2$. We will assume Conjecture 1.1.

Notation 2.1. For $A \in H^{2}(X, \mathbb{Q})$ and $\alpha \in H_{2}(X, \mathbb{Q})$ we denote by $A \cdot \alpha \in \mathbb{Q}$ the canonical pairing, by $\check{A} \in H_{2}(X, \mathbb{Z})$ the Poincaré dual and by $A^{2}$ the number $A \cdot \check{A}$. We denote by $Q_{X}$ the quadratic form on $H_{2}(X, \mathbb{Z})$ and, for a class $\eta \in H^{2}(X, \mathbb{Q})$, by $L_{\eta}$ the linear form $\alpha \mapsto \eta \cdot \alpha$ on $H_{2}(X, \mathbb{Q})$. If there is no risk of confusion we denote by $a$ the reduction of $A \in H^{2}(X, \mathbb{Z})$ modulo 2 .

For a smooth four-manifold $X$ we denote by $\widehat{X}$ the connected sum $X \# \overline{\mathbb{P}}_{2}$ of $X$ with $\mathbb{P}_{2}$ with reversed orientation, (e.g. if $X$ is a smooth complex surface, then $\widehat{X}$ is the blowup of $X$ in a point). Let $E$ be the image of the generator of $H^{2}\left(\overline{\mathbb{P}}_{2}, \mathbb{Z}\right)$ in $H^{2}(\widehat{X}, \mathbb{Z})$. We will always identify $H_{2}(X, \mathbb{Z})$ with the kernel of $L_{E}$ on $H_{2}(\widehat{X}, \mathbb{Z})$. We write $e$ for the reduction of $E$ modulo 2 .

Let $g$ be a Riemannian metric on $X$, and $P$ an $S O(3)$ principal bundle with first Pontrjagin class $p_{1}(P)=-(N+3)$. We denote by $\Phi_{c_{1}, N}^{X, g}$ the Donaldson invariant corresponding to $P$, the metric $g$, the lift $c_{1} \in H^{2}(X, \mathbb{Z})$ of $w_{2}(P)$ and a chosen orientation of $H^{2}(X, \mathbb{Z})^{+}$. We use the conventions of e.g. [F-S] which coincide up to a power of 2 with the conventions of [Ko]. If $X$ is an algebraic surface and $H$ an ample divisor we will write $\Phi_{c_{1}, N}^{X, H}$ for the invariant with respect to the Fubini-Studi metric induced by $H$. Let $p \in H_{0}(X, \mathbb{Z})$ be the class of a point. Let $A_{N}(X)$ be the set of polynomials of weight $N$ in $H_{2}(X, \mathbb{Q}) \oplus H_{0}(X, \mathbb{Q})$, where $\alpha \in H_{2}(X, \mathbb{Q})$ has weight 1 and $p$ has weight 2 , and $A_{*}(X):=\sum_{N \geq 0} A_{N}(X)$. Then $\Phi_{c_{1}, N}^{X, g}$ is a linear $\operatorname{map} A_{N}(X) \longrightarrow \mathbb{Q}$. We put $\Phi_{c_{1}, N}^{X, g}:=0$ if $N$ is not congruent to $-c_{1}^{2}+3$ modulo 4 and

$$
\Phi_{c_{1}}^{X, g}:=\sum_{N \geq 0} \Phi_{c_{1}, N}^{X, g}: A_{*}(X) \longrightarrow \mathbb{Q}
$$




\subsection{Walls and chambers.}

Definition 2.2 (see e.g. $[\mathrm{Ko}],[\mathrm{K}-\mathrm{M}])$. Let $w \in H^{2}(X, \mathbb{Z} / 2 \mathbb{Z})$ and $N$ a nonnegative integer. Let $H^{2}(X, \mathbb{R})^{+}$be the positive cone in $H^{2}(X, \mathbb{R})$. For $\xi \in H^{2}(X, \mathbb{Z})$ let

$$
W^{\xi}:=\left\{x \in H^{2}(X, \mathbb{R})^{+} \mid \xi \cdot \check{x}=0\right\} .
$$

We shall call $W^{\xi}$ a wall of type $(w, N)$, and say that it is defined by $\xi$, if $w$ is the reduction of $\xi$ modulo $2, N+3$ is congruent to $\xi^{2}$ modulo 4 and $-(N+3) \leq \xi^{2}<0$. Note that any class $\xi \in H^{2}(X, \mathbb{Z})$ with $\xi^{2}<0$ will define a wall of type $(w, N)$ for suitable $N$ and $w$ the reduction of $\xi$ modulo 2 ; we will in this case say that $\xi$ defines a wall of type $(N)$. A chamber of type $(w, N)$ is a connected component of the complement of the walls of type $(w, N)$ in $H^{2}(X, \mathbb{R})^{+}$.

For a Riemannian metric $g$ on $X$ we denote by $\omega(g) \in H^{2}(X, \mathbb{R})^{+}$the corresponding period point. For $A_{-}, A_{+} \in H^{2}(X, \mathbb{R})$ we denote by $W_{w, N}^{X}\left(A_{-}, A_{+}\right)$the set of all $\xi \in H^{2}(X, \mathbb{Z})$ defining a wall of type $(w, N)$ with $\xi \cdot \check{A}_{-}<0<\xi \cdot \check{A}_{+}$. We put

$$
W_{w}^{X}\left(A_{-}, A_{+}\right):=\bigcup_{N \geq 0} W_{w, N}^{X}\left(A_{-}, A_{+}\right) .
$$

Theorem $2.3([\mathrm{~K}-\mathrm{M}])$. Let $c_{1} \in H^{2}(X, \mathbb{Z})$ and $w$ the reduction of $c_{1}$ modulo 2 . For all $\xi \in H^{2}(X, \mathbb{Z})$ defining a wall of type $(w, N)$ we put

$$
\varepsilon\left(c_{1}, \xi, N\right):=\left(5 N+3+\xi^{2}+\left(\xi-c_{1}\right)^{2}\right) / 4 .
$$

There exists $\delta_{\xi, N}^{X}: \operatorname{Sym}^{N}\left(H_{2}(X, \mathbb{Q})\right) \longrightarrow \mathbb{Q}$ such that for all generic metrics $g_{+}$ and $g_{-}$with $\omega\left(g_{+}\right)$and $\omega\left(g_{-}\right)$in the same connected component of $H^{2}(X, \mathbb{R})^{+}$

$$
\Phi_{c_{1}, N}^{X, g_{+}}-\Phi_{c_{1}, N}^{X, g_{-}}=\sum_{\xi \in W_{w, N}^{X}\left(\omega\left(g_{-}\right), \omega\left(g_{+}\right)\right)}(-1)^{\varepsilon\left(c_{1}, \xi, N\right)} \delta_{\xi, N}^{X} .
$$

Furthermore, if $\omega\left(g_{1}\right)=-\omega(g)$, then $\Phi_{c_{1}, N}^{X, g_{1}}=-\Phi_{c_{1}, N}^{X, g}$.

Remark 2.4. (1) Our sign conventions are different from those of $[\mathrm{K}-\mathrm{M}]$ and $[\mathrm{K}-\mathrm{L}]$. In fact the sign is chosen in order to give the leading term $L_{\xi}^{N-2 d} Q_{X}^{d}$ (with $\left.d=\left(N+3+\xi^{2}\right) / 4\right)$ of $\delta_{\xi, N}^{X}$ a positive coefficient.

(2) In the future we will assume that we have fixed the orientation of $H^{2}(X, \mathbb{R})^{+}$ and thus the connected component of $H^{2}(X, \mathbb{R})^{+}$in which the period points of the metrics lie.

(3) By Theorem 2.3 we can write $\Phi_{c_{1}, N}^{X, \mathcal{C}}:=\Phi_{c_{1}, N}^{X, g}$ for any metric $g$ with $\omega(g)$ in the chamber $\mathcal{C}$.

2.2. Blowup formulas. The blowup formulas relate the Donaldson invariants of a 4-manifold $Y$ and $\widehat{Y}=Y \# \overline{\mathbb{P}}_{2}$. In the case $b_{+}(Y)>1$, when the invariants do not depend on the chamber structure, they have been shown e.g. in $[\mathrm{O}],[\mathrm{L}]$ and in the most general form in [F-S]. In the case when $X$ is a simply connected 4-manifold with $b_{+}=1 \mathrm{I}$ learned the blowup formulas from $[\mathrm{K}-\mathrm{L}]$. They then depend on the chamber structure.

Definition 2.5 (see $[\mathrm{Ko}])$. Let $\mathcal{C} \subset H^{2}(X, \mathbb{R})^{+}$be a chamber of type $(w, N)$. A chamber $\mathcal{C}_{0} \subset H^{2}(\widehat{X}, \mathbb{R})^{+}$of type $(w, N)$ (resp. $\mathcal{C}_{e} \subset H^{2}(\widehat{X}, \mathbb{R})^{+}$of type $(w+$ $e, N+1)$ ) is said to be related to $\mathcal{C}$ if and only if $\mathcal{C}$ is contained in the closure $\overline{\mathcal{C}}_{0}$ (resp. in $\overline{\mathcal{C}}_{e}$ ). 
By $[\mathrm{T}]$ the formulas of [F-S] also hold for $X$ with $b_{+}(X)=1$; we will however only need a quite easy special case (see e.g. [Ko] and [S], ex. 11).

Theorem 2.6. Let $\mathcal{C} \subset H^{2}(X, \mathbb{R})^{+}$be a chamber of type $(w, N)$, and let $\mathcal{C}_{0} \subset$ $H^{2}(\widehat{X}, \mathbb{R})^{+}$(resp. $\left.\mathcal{C}_{e} \subset H^{2}(\widehat{X}, \mathbb{R})^{+}\right)$be related chambers of types $(w, N)$ (resp. $(w+e, N+1))$. Then for all $\alpha \in A_{N}(X)$ and $\beta \in A_{N-2}(X)$ for which both sides are defined we have

$$
\begin{aligned}
\Phi_{c_{1}, N}^{X, \mathcal{C}}(\alpha) & =\Phi_{c_{1}, N}^{\widehat{X}, \mathcal{C}_{0}}(\alpha), \\
\Phi_{c_{1}, N}^{X, \mathcal{C}}(\alpha) & =\Phi_{c_{1}+E, N+1}^{\widehat{X}, \mathcal{C}_{e}} \\
\Phi_{c_{1}, N}^{\widehat{X}, \mathcal{C}_{0}}\left(\check{E}^{2} \beta\right) & =0, \\
\Phi_{c_{1}, N}^{X, \mathcal{C}}(x \beta) & =-\Phi_{c_{1}+E, N+1}^{\widehat{X}, \mathcal{C}_{e}}\left(\check{E}^{3} \beta\right) .
\end{aligned}
$$

2.3. Extension of wall-crossing formulas. We want to extend Theorem 2.3 from $\operatorname{Sym}^{N}\left(H_{2}(X, \mathbb{Q})\right)$ to $A_{N}(X)$. For this we have to extend the definition of $\delta_{\xi, N}^{X}$. In the case that $\xi$ is divisible by 2 (i.e. $w=0$ ) we also have to extend the definition of $\Phi_{c_{1}, N}^{X, g}$ to classes not in the stable range. For technical reasons we also redefine the $\delta_{\xi, N}^{X}$ in case the intersection form on $H_{2}(X, \mathbb{Z})$ is even or the rank of $H_{2}(X, \mathbb{Z})$ is at most 2 . It should not be difficult to prove that this definition agrees with that of $[\mathrm{K}-\mathrm{M}]$, but we only need that Theorem 2.3 still holds.

Definition 2.7. (1) Let $N=4 c_{2}-3$ for $c_{2} \in \mathbb{Z}$. Let $\mathcal{C}$ be a chamber of type $(0, N)$ in $H^{2}(\widehat{X}, \mathbb{R})^{+}$and $\mathcal{C}_{e}$ a related chamber of type $(e, N+1)$ on $\widehat{X}$. Then we put for all $\alpha \in A_{N}(X)$

$$
\Phi_{0, N}^{X, \mathcal{C}}(\alpha):=\Phi_{E, N+1}^{\widehat{X}, \mathcal{C}_{e}}(\check{E} \alpha) .
$$

Note that $(1)_{b}$ above guarantees that our definition restricts to the standard definition if $\alpha$ is in the stable range.

(2) Let $\xi \in H^{2}(X, \mathbb{Z})$ with $\xi^{2}<0$. We extend the definition of $\delta_{\xi, N}^{X}$ by putting $\delta_{\xi, N}^{X}:=0$ if $\xi$ does not define a wall of type $(N)$ (i.e. if $\xi^{2}$ is not congruent to $N+3$ modulo 4 or $\left.N+3+\xi^{2}<0\right)$.

(3) Assume now that the intersection form on $H_{2}(X, \mathbb{Z})$ is even or the rank of $H_{2}(X, \mathbb{Z})$ is at most 2 or that $\xi$ is divisible by 2 in $H^{2}(X, \mathbb{Z})$. Then we put for $\alpha \in \operatorname{Sym}^{N}\left(H_{2}(X, \mathbb{Q})\right)$

$$
\delta_{\xi, N}^{X}(\alpha):=\sum_{n \in \mathbb{Z}}(-1)^{n-1} \delta_{\xi+(2 n+1) E, N+1}^{\widehat{X}}(\check{E} \alpha) .
$$

Note that by (2) the sum runs in fact only through integers $n$ with $(2 n+1)^{2} \leq$ $N+4+\xi^{2}$.

(4) Assume that $\delta_{\eta, N}^{Y}\left(p^{r} \beta\right)$ is already defined for all $m$ for $Y=X \# m \overline{\mathbb{P}}_{2}$ for all $N$, all $\xi \in H^{2}(Y, \mathbb{Z})$ with $\xi^{2}<0$ and all $\beta \in \operatorname{Sym}^{N-2 r}\left(H_{2}(Y, \mathbb{Q})\right)$. Then we put

$$
\delta_{\xi, N}^{Y}\left(p^{r+1} \alpha\right):=\sum_{n \in \mathbb{Z}}(-1)^{n} \delta_{\xi+(2 n+1) E, N+1}^{\widehat{Y}}\left(\check{E}^{3} p^{r} \alpha\right)
$$

for all $\alpha \in \operatorname{Sym}^{N-2 r-2}\left(H_{2}(Y, \mathbb{Q})\right)$. Again by (2) the sum runs only through $n$ with $(2 n+1)^{2} \leq N+4+\xi^{2}$.

We note that by definition $\delta_{\xi, N}^{X}=0$ if $\xi$ does not define a wall of type $(N)$. 
Finally we put

$$
\delta_{\xi}^{X}:=\sum_{N \geq 0} \delta_{\xi, N}^{X}: A_{*}(X) \longrightarrow \mathbb{Q} .
$$

If $t$ is an indeterminate, we write $\delta_{\xi}^{X}\left(\sum_{N} \alpha_{N} t^{N}\right)$ for $\sum_{N} \delta_{\xi, N}^{X}\left(\alpha_{N}\right) t^{N}$, and similarly for $\Phi_{c_{1}}^{X, g}$.

Remark 2.8. There is a small subtlety about the definition of $\Phi_{0, N}^{X, \mathcal{C}}$ in (1). If $w \neq 0$, then, given a chamber $\mathcal{C}$ in $H^{2}(X, \mathbb{R})^{+}$of type $(w, N)$, there is a unique related chamber $\mathcal{C}_{e}$ in $H^{2}(X, \mathbb{R})^{+}$of type $(w+e, N+1)$ consisting of all $\mu+a E$ with $\mu \in \mathcal{C}$ and $a \in \mathbb{R}$ sufficiently small. If $w=0$, however, $E$ defines a wall of type $(e, N+1)$ separating two chambers $\mathcal{C}_{e}^{+}$(corresponding to $a>0$ ) and $\mathcal{C}_{e}^{-}$(corresponding to $a<0)$ of type $(e, N+1)$, which are both related to $\mathcal{C} . \Phi_{0, N}^{X, \mathcal{C}}$ is still well-defined, as $\delta_{(2 n+1) E, N+1}^{\widehat{X}}\left(\check{E}^{2 k+1} \alpha\right)=0$ for $N$ congruent to 1 modulo 4: By Conjecture 1.1 (and its extension Remark 4.3 below to $\left.A_{N}(X)\right) \delta_{E, N+1}^{\widehat{X}}\left(p^{r} \bullet\right)$ is a polynomial in $L_{E}$ and $Q_{\widehat{X}}, N+1$ is even and $E \cdot \alpha=0$.

Similarly, if $w \neq 0$, then there is a unique related chamber $\mathcal{C}_{0}$ in $H^{2}(X, \mathbb{R})^{+}$of type $(w, N)$. If $w=0$, then there are two related chambers separated by a wall defined by $2 E$, but $\delta_{2 n E, N}^{\widehat{X}}\left(\check{E}^{2 k} \alpha\right)=0$.

2.4. Vanishing on rational ruled surfaces. An important role both in the proof of the main theorem and in the application to Donaldson invariants of the projective plane is played by the following elementary vanishing result. Let $S$ be a rational ruled surface, and let $F, E \in H^{2}(S, \mathbb{Z})$ be the classes of a fibre of the projection to $\mathbb{P}_{1}$ and a section respectively. For an ample divisor $H$ let $M_{H}^{S}\left(c_{1}, c_{2}\right)$ be the moduli space of $H$-stable torsion-free sheaves with Chern classes $\left(c_{1}, c_{2}\right)$.

Lemma 2.9 ([Q2]). Assume $c_{1} \cdot F=1$, then $M_{F+\epsilon E}^{S}\left(c_{1}, c_{2}\right)$ is empty for all sufficiently small $\epsilon>0$. In particular, given $N \geq 0$, we get $\Phi_{c_{1}, N}^{S, F+\epsilon E}=0$ for all sufficiently small $\epsilon>0$.

\section{MAIN THEOREM}

We want to express the wall-crossing formulas in terms of the $q$-development of certain modular forms. We start by reminding the reader of some notations and elementary facts (see e.g. [H-B-J], $[\mathrm{R}]$ ).

Notation 3.1. Let $\mathbb{H}=\{\tau \in \mathbb{C} \mid \operatorname{Im}(\tau)>0\}$ be the complex upper half plane. We denote $q=e^{2 \pi i \tau}$ and $q^{1 / n}=e^{2 \pi i \tau / n}$. For a positive integer $n$ let

$$
\sigma_{k}(n):=\sum_{d \mid n} d^{k} \quad \text { and } \quad \sigma_{k}^{\text {odd }}(n):=\sum_{d \mid n, \quad d \text { odd }} d^{k} .
$$

Let $\eta(\tau):=q^{1 / 24} \prod_{n>0}\left(1-q^{n}\right)$ be the Dirichlet eta-function, and let $\Delta(\tau)=\eta(\tau)^{24}$ be the discriminant. We denote by $\theta(\tau):=\sum_{n \in \mathbb{Z}} q^{n^{2}}$ the theta function for the lattice $\mathbb{Z}$. We also have the Eisenstein series

$$
G_{2 k}(\tau):=-B_{k} / 2 k+\sum_{n \geq 1} \sigma_{k-1}(n) q^{n},
$$

where $B_{k}$ is the $k$-th Bernoulli number, and the 2-division value

$$
e_{3}(\tau):=1 / 12+2 \sum_{n \geq 1}(-1)^{n} \sigma_{1}^{o d d}(n) q^{n / 2} .
$$


We put $f(\tau):=\eta(2 \tau)^{3} / \theta(\tau)$. Then $\eta(2 \tau), \theta(\tau), G_{2 k}(2 \tau)$ for $k>1, e_{3}(2 \tau)$ and $f(\tau)$ are modular forms of weights $1 / 2,1 / 2,2 k, 2$ and 1 respectively for certain subgroups of $S L(2, \mathbb{Z})$, whereas $G_{2}(2 \tau)$ is only a quasimodular form (see [K-Z]).

We will denote $d \log _{q}(g):=g^{-1} d g / d q$. Note that

$$
d \log _{q}\left(g_{1} g_{2}\right)=d \log _{q}\left(g_{1}\right)+d \log _{q}\left(g_{2}\right) .
$$

Remark 3.2. We will use the following identities:

$$
\begin{aligned}
& \eta(2 \tau)^{3}=\sum_{n \in \mathbb{Z}}(-1)^{n}(n+1 / 2) q^{(n+1 / 2)^{2}}, \\
& \theta(\tau)=\frac{\eta(2 \tau)^{5}}{\eta(\tau)^{2} \eta(4 \tau)^{2}}, \quad \text { (3) } f(\tau)=\frac{\eta(\tau)^{2} \eta(4 \tau)^{2}}{\eta(2 \tau)^{2}} \text {, } \\
& q d \log _{q}(\eta(2 \tau))=-2 G_{2}(2 \tau), \\
& \text { (5) } q d \log _{q}(\theta(\tau))=-2 G_{2}(2 \tau)-e_{3}(2 \tau) \text {. }
\end{aligned}
$$

Proof. (1) and (2) are standard facts, following e.g. from the Jacobi identity. (3) follows from (2). (4) follows by an easy calculation using $(*)$, and, using also (2), the proof of (5) is similar.

The main result of this paper is the following.

Theorem 3.3. Let $X$ be a simply connected 4 -manifold with $b_{+}=1$ and signature $\sigma(X)$. Let $\xi \in H^{2}(X, \mathbb{Z})$ with $\xi^{2}<0$. For $\alpha \in H_{2}(X, \mathbb{Z})$ denote

$$
\begin{aligned}
g_{\xi}^{X}(\alpha z, x, \tau):=\exp \left(\frac{(\xi \cdot \alpha) z}{2 f(\tau)}-Q_{X}(\alpha) \frac{z^{2}\left(G_{2}(2 \tau)+e_{3}(2 \tau) / 2\right)}{f(\tau)^{2}}+3 x \frac{e_{3}(2 \tau)}{f(\tau)^{2}}\right) \\
\cdot \theta(\tau)^{\sigma(X)} f(\tau) \frac{\Delta(2 \tau)^{2}}{\Delta(\tau) \Delta(4 \tau)} .
\end{aligned}
$$

Then

$$
\delta_{\xi}^{X}(\exp (\alpha z+p x))=\operatorname{res}_{q=0}\left(q^{-\xi^{2} / 4} g_{\xi}^{X}(\alpha z, x, \tau) d q / q\right) .
$$

Remark 3.4. (1) One can see that this expression for $\delta_{\xi}^{X}$ is not compatible with the simple type condition. In particular, given $c \in H^{2}(X, \mathbb{Z})$, a 4-manifold $X$ with $b_{+}=1$ will be of $c$-simple type at most for some special points in the closure $\bar{C}_{X}$ of the positive cone of $X$. It had already been shown in [K-L] that $\mathbb{P}_{2}$ is not of simple type and that there is no chamber for which $\mathbb{P}_{1} \times \mathbb{P}_{1}$ is of simple type. It is easy to see from this that rational algebraic surfaces $X$ can be of simple type at most for special points in $\bar{C}_{X}$.

(2) The expression $\operatorname{res}_{q=0}\left(q^{-\xi^{2} / 4} g_{\xi}^{X}(\alpha z, x, \tau) d q / q\right)$ is just the coefficient of $q^{\xi^{2} / 4}$ of $g_{\xi}^{X}(\alpha z, x, \tau)$. The current formulation is however more intrinsic. Note also that $d q / q=2 \pi i d \tau$.

(3) We see that the coefficient $g_{N-2 r, r}$ of $z^{N-2 r} x^{r}$ in $g_{\xi}^{X}(\alpha z, x, \tau)$ is $q^{-(N+3) / 4}$ multiplied by a power series in $q$. In particular, if $\xi$ defines a wall of type $(N)$, then $q^{-\xi^{2} / 4} g_{N-2 r, r}$ is a Laurent series in $q$. If $\xi$ with $\xi^{2}<0$ does not define a wall of type $(N)$, then the constant term of $q^{-\xi^{2} / 4} g_{N-2 r, r}$ is zero.

(4) It would be interesting to know whether for classes $\xi \in H^{2}(X, \mathbb{Z})$ with $\xi^{2} \geq$ 0 the expression $\operatorname{res}_{q=0}\left(q^{-\xi^{2} / 4} g_{\xi}^{X}(\alpha z, x, \tau) d q / q\right)$ has a geometrical or gaugetheoretical meaning.

As a reasonably straightforward application of Theorem 3.3 we can determine all the Donaldson invariants of the projective plane $\mathbb{P}_{2}$. 
Theorem 3.5. We denote by $\sqrt{i}$ a primitive 8-th root of unity and by $H$ the hyperplane class in $H^{2}\left(\mathbb{P}_{2}, \mathbb{Z}\right)$. Put

$$
e_{n}(z, x, \tau):=\exp \left(\frac{n}{2} \frac{\sqrt{i} z}{f(\tau)}-i z^{2} \frac{G_{2}(2 \tau)+e_{3}(2 \tau) / 2}{f(\tau)^{2}}+3 i x \frac{e_{3}(2 \tau)}{f(\tau)^{2}}\right) \frac{\Delta(2 \tau)^{2}}{\Delta(\tau) \Delta(4 \tau)} .
$$

Then

(1)

$$
\Phi_{H}^{\mathbb{P}_{2}}(\exp (\check{H} z+p x))=\operatorname{res}_{q=0}\left(\sum_{\substack{n>0 \text { odd } \\ a>n \text { even }}}(-1)^{(n+1) / 2} q^{\left(a^{2}-n^{2}\right) / 4} e_{n}(z, x, \tau) f(\tau)\right) \frac{d q}{q} .
$$

$$
\Phi_{0}^{\mathbb{P}_{2}}(\exp (\check{H} z+p x))=\operatorname{res}_{q=0}\left(\sum_{\substack{n>0 \text { even } \\ a>n \text { odd }}}(-1)^{(a-1) / 2} q^{\left(a^{2}-n^{2}\right) / 4} \frac{a}{2 \sqrt{i}} e_{n}(z, x, \tau)\right) \frac{d q}{q} .
$$

In (2) we have used Definition 2.7 to define $\Phi_{0}^{\mathbb{P}_{2}}\left(\check{H}^{N-2 r} p^{r}\right)$ for $r \geq(N-5) / 4$. One can check that (up to different sign conventions) (1) and (2) agree with the explicit computations in $[\mathrm{K}-\mathrm{L}]$ and $[\mathrm{E}-\mathrm{G} 2]$.

Proof of Theorem 3.5 from Theorem 3.3. Let $Y$ be the blowup of $\mathbb{P}_{2}$ in a point, and let $E \in H^{2}(Y, \mathbb{Z})$ be the class of the exceptional divisor. Let $F=H-E$ be the class of a fibre of the ruling $Y \longrightarrow \mathbb{P}_{1}$. Fix a nonnegative integer $N$. By Lemma 2.9 we get for $\epsilon>0$ sufficiently small $\Phi_{H, N}^{Y, F+\epsilon E}=0=\Phi_{E, N+1}^{Y, F+\epsilon E}$. On the other hand the chamber of $H-\epsilon E$ is related to the polarization $H$ of $\mathbb{P}_{2}$. Thus we obtain by the blowup formulas $(0)_{b}$ and $(1)_{b}$ that $\Phi_{H, N}^{\mathbb{P}_{2}}=\Phi_{H, N}^{Y, H-\epsilon E}$ and $\Phi_{0, N}^{\mathbb{P}_{2}}=\Phi_{E, N+1}^{Y, H-\epsilon E}$. So we get by Theorem 2.3 (and Lemma 4.1 below) the formulas

$$
\begin{aligned}
& \Phi_{H}^{\mathbb{P}_{2}}(\exp (\check{H} z+p x))=\sum_{\xi \in W_{h}^{Y}(F, H)} \sqrt{i}^{\left(\xi^{2}+3\right)+(\xi-H)^{2}} \delta_{\xi}^{Y}(\exp (-\sqrt{i} \check{H} z+i p x)), \\
& \Phi_{0}^{\mathbb{P}_{2}}(\exp (\check{H} z+p x))=\sum_{\xi \in W_{e}^{Y}(F, H)} \sqrt{i}^{\left(\xi^{2}+3\right)+(\xi-E)^{2}} \delta_{\xi}^{Y}(-\sqrt{i} \check{E} \exp (-\sqrt{i} \check{H} z+i p x)) .
\end{aligned}
$$

It is easy to see that

$$
\begin{aligned}
& W_{h}^{Y}(F, H)=\left\{(2 n-1) H-2 a E \mid a \geq n \in \mathbb{Z}_{>0}\right\}, \\
& W_{e}^{Y}(F, H)=\left\{2 n H-(2 a-1) E \mid a>n \in \mathbb{Z}_{>0}\right\} .
\end{aligned}
$$

For $\xi=n H-a E$ we get $-\xi^{2} / 4=\left(a^{2}-n^{2}\right) / 4$. Furthermore $\sqrt{i}^{-\left(\xi^{2}+3\right)+(\xi-H)^{2}}=$ $(-1)^{(n+1) / 2}$ if $n$ is odd and $a$ is even, and $\sqrt{i}^{\left(\xi^{2}+3\right)+(\xi-E)^{2}}=i^{a+2}$ if $n$ is even and $a$ is odd. Thus, replacing $-\sqrt{i}$ by $\sqrt{i}$, (1) follows directly by applying Theorem 3.3 .

(2) follows the same way using that

$$
\begin{gathered}
\delta_{\xi}^{Y}(-\sqrt{i} \check{E} \exp (-\sqrt{i} \check{H} z+i p x))=\left.\frac{d}{d w}\left(\delta_{\xi}^{Y}(\exp (-\sqrt{i}(\check{E} w+\check{H} z)+i p x))\right)\right|_{w=0} \\
=\operatorname{res}_{q=0}\left(\left.q^{-\xi^{2} / 4} \frac{d}{d w}\left(g_{\xi}^{\widehat{\mathbb{P}}_{2}}(-\sqrt{i}(\check{E} w+\check{H} z), i x, \tau)\right)\right|_{w=0}\right) .
\end{gathered}
$$


Remark 3.6. The arguments in section 6 of [E-G2] show that, using Theorem 3.3 and the blowup formulas, we can get explicit generating functions for all the Donaldson invariants of all rational surfaces $S$ in all chambers of $H^{2}(S, \mathbb{R})^{+}$. In $[\mathrm{K}-\mathrm{L}]$ it had been shown (also using the blowup formulas) that the wall-crossing terms on $\mathbb{P}_{2} \# 2 \overline{\mathbb{P}}_{2}$ determine the Donaldson invariants on $\mathbb{P}_{2}$ and $\mathbb{P}_{1} \times \mathbb{P}_{1}$.

\section{Proof of the main theorem}

We give a brief outline of the argument. Let $\xi \in H^{2}(X, \mathbb{Z})$ define a wall of type $(w, N)$, and let $\mathcal{C}_{-}$and $\mathcal{C}_{+}$be the two chambers separated by $W^{\xi}$. The related chambers $\mathcal{C}_{-0}$ and $\mathcal{C}_{+0}$ of type $(w, N)$ (resp. $\mathcal{C}_{-e}$ and $\mathcal{C}_{+e}$ of type $\left.(w+e, N+1)\right)$ on $\widehat{X}$ are now separated by several walls. We can express $\delta_{\xi, N}^{X}$ by first applying the blowup formula to the pair $\mathcal{C}_{-}, \mathcal{C}_{-0}$ of related chambers, then summing up the wall-crossing formulas for all walls between $\mathcal{C}_{-0}$ and $\mathcal{C}_{+0}$ and finally applying again the blowup formula for $\mathcal{C}_{+}, \mathcal{C}_{+0}$ (and similarly for $\mathcal{C}_{-e}, \mathcal{C}_{+e}$ ). The blowup formulas $(0)_{b}-(3)_{b}$ from 2.6 will give relations $(0)_{r}-(3)_{r}$ between the $\delta_{\xi, N}^{X}$ and the $\delta_{\xi, N}^{\widehat{X}}$. Using Conjecture 1.1 we encode this information (for all blowups of $X$ ) in a suitable generating function $\Lambda_{X}$ in several variables. Then we can translate $(0)_{r}-(3)_{r}$ into differential equations $(0)_{d}-(3)_{d}$ for $\Lambda_{X}$, which determine $\Lambda_{X}$ up to multiplication by a function $\lambda_{X}(\tau)$. We finally determine $\lambda_{X}(\tau)$ by specializing to the case $X=\mathbb{P}_{1} \times \mathbb{P}_{1}$ and applying Lemma 2.9.

Lemma 4.1. Let $w$ be the reduction modulo 2 of $c_{1} \in H^{2}(X, \mathbb{Z})$, and let $N$ be a nonnegative integer. Let $g_{-}$and $g_{+}$be two metrics on $X$, whose period points $\omega\left(g_{-}\right)$ and $\omega\left(g_{+}\right)$do not lie on a wall of type $(w, N)$. Let

$$
W:=W_{w, N}^{X}\left(\omega\left(g_{-}\right), \omega\left(g_{+}\right)\right) .
$$

Then we have for all $\alpha \in A_{N}(X)$ and $\beta \in A_{N-2}(X)$ :

(a) $\Phi_{c_{1}, N}^{X, g_{+}}(\alpha)-\Phi_{c_{1}, N}^{X, g_{-}}(\alpha)=\sum_{\xi \in W}(-1)^{\varepsilon\left(c_{1}, \xi, N\right)} \delta_{\xi, N}^{X}(\alpha)$,

$(0)_{r} \quad \Phi_{c_{1}, N}^{X, g_{+}}(\alpha)-\Phi_{c_{1}, N}^{X, g_{-}}(\alpha)=\sum_{\xi \in W}(-1)^{\varepsilon\left(c_{1}, \xi, N\right)} \sum_{n \in \mathbb{Z}} \delta_{\xi+2 n E}^{\widehat{X}}(\alpha)$, $\Phi_{c_{1}, N}^{X, g_{+}}(\alpha)-\Phi_{c_{1}, N}^{X, g_{-}}(\alpha)=\sum_{\xi \in W}(-1)^{\varepsilon\left(c_{1}, \xi, N\right)} \sum_{n \in \mathbb{Z}}(-1)^{n-1} \delta_{\xi+(2 n+1) E, N+1}^{\widehat{X}}(\check{E} \alpha)$,

$$
0=\sum_{\xi \in W}(-1)^{\varepsilon\left(c_{1}, \xi, N\right)} \sum_{n \in \mathbb{Z}} \delta_{\xi+2 n E, N}^{\widehat{X}}\left(\check{E}^{2} \beta\right),
$$

$$
\Phi_{c_{1}, N}^{X, g_{+}}(p \beta)-\Phi_{c_{1}, N}^{X, g_{-}}(p \beta)=\sum_{\xi \in W}(-1)^{\varepsilon\left(c_{1}, \xi, N\right)} \sum_{n \in \mathbb{Z}}(-1)^{n} \delta_{\xi+(2 n+1) E, N+1}^{\widehat{X}}\left(\check{E}^{3} \beta\right) .
$$

(a) says that Theorem 2.3 extends to our definition of $\delta_{\xi, N}^{X}$.

Proof. We assume that $N$ is congruent to $-\left(c_{1}^{2}+3\right)$ modulo 4 (otherwise both sides of $(a),(0)_{r}-(3)_{r}$ are trivially zero). Let $\mathcal{C}_{-}$and $\mathcal{C}_{+}$be the chambers of type $(w, N)$ of $\omega\left(g_{-}\right)$and $\omega\left(g_{+}\right)$respectively. Let $\mathcal{C}_{-0}$ and $\mathcal{C}_{+0}\left(\right.$ resp. $\mathcal{C}_{-e}$ and $\left.\mathcal{C}_{+e}\right)$ be related chambers in $H^{2}(\widehat{X}, \mathbb{R})^{+}$of type $(w, N)($ resp. $(w+e, N+1))$. 
Claim.

$$
\begin{aligned}
W_{w, N}^{\widehat{X}}\left(\mathcal{C}_{-0}, \mathcal{C}_{+0}\right) & =\left\{\xi+2 n E \mid \xi \in W, n \in \mathbb{Z}, n^{2} \leq\left(N+3+\xi^{2}\right) / 4\right\}, \\
W_{w+e, N+1}^{\widehat{X}}\left(\mathcal{C}_{-e}, \mathcal{C}_{+e}\right) & =\left\{\xi+(2 n+1) E \mid \xi \in W, n \in \mathbb{Z},(2 n+1)^{2} \leq N+4+\xi^{2}\right\} .
\end{aligned}
$$

In the case that $w=0$, we assume that $\mathcal{C}_{-0}$ and $\mathcal{C}_{+0}$ (resp. $\mathcal{C}_{-e}$ and $\mathcal{C}_{+e}$ ) lie on the same side of $W^{2 E}$ (resp. $W^{E}$ ). The claim is essentially obvious: Any $\eta \in W_{w, N}^{\widehat{X}}\left(\mathcal{C}_{-0}, \mathcal{C}_{+0}\right)$ must be of the form $\xi+\alpha E$ for $\xi \in W$. By the definition of a wall we see that $\alpha$ must be an even integer $2 n$ with $n^{2} \leq\left(N+3+\xi^{2}\right) / 4$. On the other hand it is obvious that all $\xi+2 n E$ with $\xi \in W$ and $n^{2} \leq\left(N+3+\xi^{2}\right) / 4$ lie in $W_{w, N}^{\widehat{X}}\left(\mathcal{C}_{-0}, \mathcal{C}_{+0}\right)$. For $W_{w+e, N+1}^{\widehat{X}}\left(\mathcal{C}_{-e}, \mathcal{C}_{+e}\right)$ we argue analogously.

Using this description of $W_{w, N}^{\widehat{X}}\left(\mathcal{C}_{-0}, \mathcal{C}_{+0}\right)$ and $W_{w+e, N+1}^{\widehat{X}}\left(\mathcal{C}_{-e}, \mathcal{C}_{+e}\right)$, we see that for $X$ with $b_{2}(X)>2$ and odd intersection form and $\alpha \in \operatorname{Sym}^{N}\left(H^{2}(X, \mathbb{Q})\right)$ and $\beta \in$ $\operatorname{Sym}^{N-2}\left(H^{2}(X, \mathbb{Q})\right)$, the formulas $(0)_{r}-(3)_{r}$ are just straightforward translations of $(0)_{b}-(3)_{b}$ (note that $\varepsilon\left(c_{1}, \xi, N\right)-\varepsilon\left(c_{1}+E, \xi+(2 n+1) E, N+1\right)$ is congruent to $n-1$ modulo 2 ).

Now assume that the intersection form of $X$ is even or $b_{2}(X) \leq 2$ or $\xi$ is divisible by 2 in $H^{2}(X, \mathbb{Z})$. Then Definition 2.7, the description of $W_{w, N}^{\widehat{X}}\left(\mathcal{C}_{-e}, \mathcal{C}_{+e}\right)$ and $(1)_{b}$ imply immediately that $(a)$ holds for all $\alpha \in \operatorname{Sym}^{N}\left(H^{2}(X, \mathbb{Q})\right)$. We show $(0)_{r}-(3)_{r}$ for $\alpha \in \operatorname{Sym}^{N}\left(H^{2}(X, \mathbb{Q})\right)$ (we only carry out the case of $(0)_{r}$, the other cases are analogous). Let $\widetilde{X}:=\widehat{X} \# \overline{\mathbb{P}}_{2}$; we denote by $F$ the generator of $H^{2}\left(\overline{\mathbb{P}}_{2}, \mathbb{Z}\right)$. Then by Definition 2.7 and $(0)_{r}$ for $\widehat{X}$ we get

$$
\begin{aligned}
\Phi_{c_{1}, N}^{X, \mathcal{C}_{+}}(\alpha)-\Phi_{c_{1}, N}^{X, \mathcal{C}_{-}}(\alpha) & =\sum_{\xi \in W} \sum_{m \in \mathbb{Z}}(-1)^{m+1} \delta_{\xi+(2 m+1) E, N+1}^{\widehat{X}}(\check{E} \alpha) \\
& =\sum_{\xi \in W} \sum_{n \in \mathbb{Z}} \sum_{m \in \mathbb{Z}}(-1)^{m+1} \delta_{\xi+2 n F+(2 m+1) E, N+1}^{\widetilde{X}}(\check{E} \alpha) \\
& =\sum_{\xi \in W} \sum_{n \in \mathbb{Z}} \delta_{\xi+2 n E, N}^{\widehat{X}}(\alpha) .
\end{aligned}
$$

Now let $X$ be general. We assume $(a),(0)_{r}-(3)_{r}$ for all blowups $Y$ of $X$ and all classes $\alpha=p^{l} \beta$ with $\beta \in \operatorname{Sym}^{k}\left(H_{2}(Y, \mathbb{Q})\right)$ for some $k$. Then $(3)_{r}$ implies immediately $(a)$ for $p \alpha$. The proof of $(0)_{r}-(3)_{r}$ for $p \alpha$ is analogous to the last section. We only carry out the case of $(1)_{r}$. Let $\tilde{Y}:=\widehat{Y} \# \overline{\mathbb{P}}_{2}$; we denote by $F$ the generator of $H^{2}\left(\overline{\mathbb{P}}_{2}, \mathbb{Z}\right)$. We get by Definition 2.7

$$
\begin{aligned}
\Phi_{c_{1}, N}^{Y, \mathcal{C}_{+}}(p \alpha) & -\Phi_{c_{1}, N}^{Y, \mathcal{C}_{-}}(p \alpha)=\sum_{\xi \in W} \sum_{n \in \mathbb{Z}}(-1)^{n} \delta_{\xi+(2 n+1) E, N+1}^{\widehat{Y}}\left(\check{E}^{3} \alpha\right) \\
& =\sum_{\xi \in W} \sum_{n \in \mathbb{Z}} \sum_{m \in \mathbb{Z}}(-1)^{n}(-1)^{m-1} \delta_{\xi+(2 n+1) E+(2 m+1) F, N+2}^{\tilde{Y}}\left(\check{F} \check{E}^{3} \alpha\right) \\
& =\sum_{\xi \in W} \sum_{m \in \mathbb{Z}}(-1)^{m-1} \delta_{\xi+(2 m+1) E, N+1}^{\widehat{Y}}(\check{E} p \alpha) .
\end{aligned}
$$

Lemma 4.2. For $\xi \in H^{2}(X, \mathbb{Z})$ we get

$$
\exp \left(L_{(\xi+n E) / 2}+Q_{\widehat{X}}\right)\left(\check{E}^{k} \bullet\right)=\sum_{s+2 t=k}(n / 2)^{s}(-1)^{s+t} \frac{k !}{s ! t !} \exp \left(L_{\xi / 2}+Q_{X}\right),
$$


as a map $\sum_{N \geq 0} \operatorname{Sym}^{N}\left(H_{2}(X, \mathbb{Q})\right) \longrightarrow \mathbb{Q}$.

Proof.

$$
\exp \left(L_{(\xi+n E) / 2}+Q_{\widehat{X}}\right)\left(\check{E}^{k} \bullet\right)=\left.\frac{d^{k}}{d w^{k}} \exp \left(\left(L_{\xi}-n w\right) / 2+Q_{X}-w^{2}\right)\right|_{w=0},
$$

and the result follows by induction.

Remark 4.3. Using Definition 2.7, Lemma 4.2 and easy induction we see that Conjecture 1.1 implies that $\delta_{\xi, N}^{X}\left(p^{r} \bullet\right)$ is a polynomial in $L_{\xi / 2}$ and $Q_{X}$ with coefficients only depending on $N, \xi^{2}, r$ and the homotopy type of $X$.

Definition 4.4. For all $b \geq 0$ let $X(b):=X \# b \overline{\mathbb{P}}_{2}$. Let $l, k, r, b \in \mathbb{Z}$, put $N:=$ $l+2 k+2 r$, and assume that there exists a class $\xi \in H^{2}(X(b), \mathbb{Z})$ with $w=\xi^{2} / 4<0$. Then we put

$$
P(l, k, r, b, w):=\frac{l ! k !}{(l+2 k) !} \operatorname{Coeff}_{L_{\xi / 2}^{l} Q_{X(b)}^{k}} \delta_{\xi, N}^{X(b)}\left(p^{r} \bullet\right) .
$$

(By definition $P(l, k, r, b, w)$ will be zero if $\xi$ does not define a wall of type $(N)$ or if one of $l, k, r, b$ is negative.) Note that $P(l, k, r, b, w)$ is well defined: By Conjecture 1.1 and Remark 4.3, $\delta_{\xi, N}^{X(b)}\left(p^{r} \bullet\right)$ is a polynomial in $L_{\xi / 2}$ and $Q_{X(b)}$. As $b_{2}(X)>1$, the monomials $L_{\xi / 2}^{l} Q_{X(b)}^{k}$ are linearly independent as linear maps $\operatorname{Sym}^{l+2 k}\left(H_{2}(X, \mathbb{Q})\right) \longrightarrow \mathbb{Q}$, therefore the coefficients of $L_{\xi / 2}^{l} Q_{X(b)}^{k}$ in $\delta_{\xi, N}^{X(b)}\left(p^{r} \bullet\right)$ are well defined. Finally, again by Conjecture 1.1 they depend only on the numbers $l, k, r, b, w$.

Lemma 4.5. For all $(l, k, r, b, w)$ with $b \geq 0$, if the left hand side of the equations below is well defined, then the right hand side is also, and

$$
\begin{aligned}
&(0)_{s} \quad P(l, k, r, b, w)=\sum_{n \in \mathbb{Z}} P\left(l, k, r, b+1, w-n^{2}\right), \\
&(1)_{s} \quad P(l, k, r, b, w)= \sum_{n \in \mathbb{Z}}(-1)^{n}(n+1 / 2) P\left(l+1, k, r, b+1, w-(n+1 / 2)^{2}\right), \\
&(2)_{s} \sum_{n \in \mathbb{Z}} n^{2} P\left(l, k, r, b+1, w-n^{2}\right)=2 \sum_{n \in \mathbb{Z}} P\left(l-2, k+1, r, b+1, w-n^{2}\right), \\
&(3)_{s} \\
& P(l, k, r+1, b, w)= \sum_{n \in \mathbb{Z}}(-1)^{n+1}\left((n+1 / 2)^{3} P\left(l+3, k, r, b+1, w-(n+1 / 2)^{2}\right)\right. \\
&\left.-6(n+1 / 2) P\left(l+1, k+1, r, b+1, w-(n+1 / 2)^{2}\right)\right) .
\end{aligned}
$$

Proof. Take $(l, k, r, b, w)$ such that there exists a class $\xi \in H^{2}(X(b), \mathbb{Z})$ with $w:=$ $\xi^{2} / 4<0$. Let $N:=l+2 k+2 r$. We can assume that $\xi$ defines a wall of type $(N)$ (otherwise both sides of $(0)_{s}-(3)_{s}$ are trivially zero).

Assume first that $b_{2}(X)>2$ and that in addition the intersection form on $H_{2}(X, \mathbb{Z})$ is odd, or $b>0$. Then we can find an $\eta$ which is not divisible in $H^{2}(X(b), \mathbb{Z})$ with $\eta^{2}=\xi^{2}$. (The intersection form is $(1) \oplus(-1)^{\oplus b_{2}(X(b))-1}$, therefore we can find orthogonal classes $h, e_{1}, e_{2}$ with $Q_{X(b)}(h)=1=-Q_{X(b)}\left(e_{1}\right)=$ $-Q_{X(b)}\left(e_{2}\right)$, and we put $\eta:=n h+(n+1) e_{1}\left(\right.$ resp. $\left.\eta:=n h+(n+1) e_{1}+e_{2}\right)$ if $\xi^{2}=-(2 n+1)$ (resp. $\xi^{2}=-(2 n+2)$ ) for $n \in \mathbb{Z}_{>0}$.) We can therefore assume that $\xi$ is not divisible in $H^{2}(X(b), \mathbb{Z})$. Let $\mathcal{C}_{-}$and $\mathcal{C}_{+}$be the two chambers 
separated by $W^{\xi}$, with $\xi \cdot \check{a}_{-}<0<\xi \cdot \check{a}_{+}$for $a_{-} \in \mathcal{C}_{-}$and $a_{+} \in \mathcal{C}_{+}$. Assume that $N+3+4 \xi^{2}<0$. Then $W_{w, N}^{X(b)}\left(a_{-}, a_{+}\right)=\{\xi\}$. Therefore we can replace $\Phi_{c_{1}, N}^{X(b), \mathcal{C}_{+}}-\Phi_{c_{1}, N}^{X(b), \mathcal{C}_{-}}$in $(0)_{r}-(3)_{r}$ by $(-1)^{\varepsilon\left(c_{1}, \xi, N\right)} \delta_{\xi, N}^{X(b)}$. Now we apply Lemma 4.2 and the definition of the $P(l, k, r, b, w)$ to obtain the result.

If $m:=N+3+4 \xi^{2} \geq 0$ we use induction on $m$. So we assume that the result is true for all $m^{\prime}<m$. Then $W_{w, N}^{X(b)}\left(a_{-}, a_{+}\right)=\{\xi\} \cup W_{m}$, where the classes $\eta \in W_{m}$ satisfy $N+3+\eta^{2}<m$. So by induction the result holds for all $\eta \in W_{m}$ and thus by Lemma 4.1 also for $\xi$.

Finally if $b_{2}(X) \leq 2$ or the intersection form on $H_{2}(X, \mathbb{Z})$ is even, and $b=0$, then we use Definition 2.7

$$
\delta_{\xi, N}^{X}(\alpha):=\sum_{n \in \mathbb{Z}}(-1)^{n-1} \delta_{\xi+(2 n+1) E, N+1}^{\widehat{X}}(\check{E} \alpha) .
$$

The result now follows by a computation analogous to the proof of Lemma 4.1 (and to the beginning of the proof of Lemma 4.8 below).

We want to use the $P(l, k, r, b, w)$ as the coefficients of a power series, which should solve a system of differential equations. This does not work directly, because at the moment we have only coefficients with $w<0$. So we have to "complete" the coefficients, i.e. to define the $P(l, k, r, b, w)$ for all $l, k, r, b, w$ by making use of relation $(1)_{s}$.

Definition 4.6. For all $l, k, r, b \in \mathbb{Z}$ and all $w \in \frac{1}{4} \mathbb{Z}$ define $P(l, k, r, b, w)$ inductively by

(1) If $w=\xi^{2} / 4<0$ for $\xi \in H^{2}(X(b), \mathbb{Z})$, then apply Definition 4.4.

(2) We put

$$
P(l, k, r, b, w):=\sum_{n \in \mathbb{Z}}(-1)^{n}(n+1 / 2) P\left(l+1, k, r, b+1, w-(n+1 / 2)^{2}\right)
$$

whenever the right hand side is already defined inductively by (1) and (2). Note that the sum is again finite.

We check that the $P(l, k, r, b, w)$ are well defined. For this we have to see (a) that (1) and (2) give the same $P(l, k, r, b, w)$ whenever both apply, but this is the contents of relation $(1)_{s}$; and (b) that the above definition determines $P(l, k, r, b, w)$ for each 5-tuple $(l, k, r, b, w) \in \mathbb{Z}^{4} \times \frac{1}{4} \mathbb{Z}$. If $w \leq 0$, then on $X(1)$ for all $n \in \mathbb{Z}$ there exist classes $\eta_{n}$ with $\eta_{n}^{2}=4 w-(2 n+1)^{2}<0$ (as the intersection form on $X(1)$ is odd and of rank $\geq 3)$, and thus $P(l, k, r, b, w)$ is defined by (2). Now assume that $P\left(l, k, r, b, w^{\prime}\right)$ is defined for all $l, k, r, b$ and all $w^{\prime}<w$. Then we use (2) again to define $P(l, k, r, b, w)$. We put

$$
\Lambda_{X}(L, Q, x, t, \tau):=\sum_{(l, k, r, b) \in \mathbb{Z}^{4}} \sum_{w \in \frac{1}{4} \mathbb{Z}} P(l, k, r, b, w) \frac{L^{l} Q^{k} x^{r} t^{b} q^{w}}{l ! k ! r ! b !},
$$

where again $\tau \in \mathbb{H}$ and $q=e^{2 \pi i \tau}$. Note that (1), (2) and Definitions 2.7 and 4.4 imply that $P(l, k, r, b, w)=0$ if $l+2 k+2 r+3$ is not congruent to $4 w$ modulo 4 .

Now $\Lambda_{X}$ encodes all the wall-crossing formulas for all blowups of $X$. 
Remark 4.7. Let $\xi \in H^{2}(X(b), \mathbb{Z})$ be a class with $\xi^{2}<0$. Then for all $\alpha \in$ $H_{2}(X(b), \mathbb{Q})$

$$
\delta_{\xi}^{X(b)}(\exp (\alpha z+p x))=\operatorname{res}_{q=0}\left(\left.\frac{\partial^{b}}{\partial t^{b}}\left(q^{-\xi^{2} / 4} \Lambda_{X}\left((\xi / 2 \cdot \alpha) z, Q_{X}(\alpha) z^{2}, x, t, \tau\right) \frac{d q}{q}\right)\right|_{t=0} .\right.
$$

Proof. This follows directly from the definition.

Lemma 4.8. $\Lambda_{X}$ satisfies the differential equations

$$
\begin{aligned}
\theta(\tau) \frac{\partial}{\partial t} \Lambda_{X} & =\Lambda_{X}, \\
\eta(2 \tau)^{3} \frac{\partial}{\partial L} \frac{\partial}{\partial t} \Lambda_{X} & =\Lambda_{X}, \\
2 \theta(\tau) \frac{\partial}{\partial Q} \Lambda_{X} & =\left(q \frac{d}{d q} \theta(\tau)\right) \frac{\partial^{2}}{\partial L^{2}} \Lambda_{X}, \\
\frac{\partial}{\partial x} \Lambda_{X} & =\left(q \frac{d}{d q} \eta(2 \tau)^{3}\right) \frac{\partial^{3}}{\partial L^{3}} \frac{\partial}{\partial t} \Lambda_{X}-6 \eta(2 \tau)^{3} \frac{\partial}{\partial L} \frac{\partial}{\partial Q} \frac{\partial}{\partial t} \Lambda_{X} .
\end{aligned}
$$

Proof. We first want to see that the relations $(0)_{s}-(3)_{s}$ hold for all $(l, k, r, b, w) \in$ $\mathbb{Z}^{4} \times \frac{1}{4} \mathbb{Z}$, i.e. that the recursive definition is compatible with $(0)_{s}-(3)_{s}$. The proof is similar in all cases, so we just do $(0)_{s}$. We assume that $(0)_{s}$ holds for all $\left(l, k, r, b, w^{\prime}\right)$ with $w^{\prime}<w$. Then we get

$$
\begin{aligned}
P(l, k, r, b, w) & =\sum_{n \in \mathbb{Z}}(-1)^{n}(n+1 / 2) P\left(l+1, k, r, b+1, w-(n+1 / 2)^{2}\right) \\
& =\sum_{n, m \in \mathbb{Z}}(-1)^{n}(n+1 / 2) P\left(l+1, k, r, b+2, w-(n+1 / 2)^{2}-m^{2}\right) \\
& =\sum_{m \in \mathbb{Z}} P\left(l+1, k, r, b+1, w-m^{2}\right) .
\end{aligned}
$$

We now translate $(0)_{s}-(3)_{s}$ into differential equations $(0)_{d}-(3)_{d}$ :

$$
\begin{aligned}
\Lambda_{X} & =\sum_{(l, k, r, b, w)} P(l, k, r, b, w) \frac{L^{l} Q^{k} x^{r} t^{b} q^{w}}{l ! k ! r ! b !} \\
& =\sum_{(l, k, r, b, w)} \sum_{n \in \mathbb{Z}} P(l, k, r, b, w) \frac{L^{l} Q^{k} x^{r} t^{b-1} q^{w+n^{2}}}{l ! k ! r !(b-1) !} \\
& =\theta(\tau) \frac{\partial}{\partial t} \Lambda_{X} .
\end{aligned}
$$

Similarly we get

$$
\begin{aligned}
\Lambda_{X} & =\sum_{(l, k, r, b, w)} \sum_{n \in \mathbb{Z}}(-1)^{n}(n+1 / 2) P(l, k, r, b, w) \frac{L^{l-1} Q^{k} x^{r} t^{b-1} q^{w+(n+1 / 2)^{2}}}{(l-1) ! k ! r !(b-1) !} \\
& =\sum_{n \in \mathbb{Z}}(-1)^{n}(n+1 / 2) q^{(n+1 / 2)^{2}} \frac{\partial}{\partial L} \frac{\partial}{\partial t} \Lambda_{X},
\end{aligned}
$$


and $(1)_{d}$ follows from Remark 3.2. Furthermore

$$
\begin{aligned}
0 & =\sum_{(l, k, r, b, w)} P(l, k, r, b, w) \sum_{n \in \mathbb{Z}}\left(\frac{n^{2} L^{l-2} Q^{k}}{(l-2) ! k !}-\frac{2 L^{l} Q^{k-1}}{l !(k-1) !}\right) \frac{x^{r} t^{b-1} q^{w+n^{2}}}{r !(b-1) !} \\
& =\left(q \frac{d}{d q} \theta(\tau)\right) \frac{\partial^{2}}{\partial L^{2}} \frac{\partial}{\partial t} \Lambda_{X}-2 \frac{\partial}{\partial Q} \frac{\partial}{\partial t} \Lambda_{X} .
\end{aligned}
$$

Finally we get

$$
\begin{aligned}
\frac{\partial}{\partial x} \Lambda_{X}= & \sum_{(l, k, r, b, w)} P(l, k, r+1, b, w) \frac{L^{l} Q^{k} x^{r} t^{b} q^{w}}{l ! k ! r ! b !} \\
= & \sum_{(l, k, r, b, w)} P(l, k, r, b, w) \sum_{n \in \mathbb{Z}}(-1)^{n+1}\left(\frac{(n+1 / 2)^{3} L^{l-3} Q^{k}}{(l-3) ! k !}\right. \\
& \left.\quad-\frac{6(n+1 / 2) L^{l-1} Q^{k-1}}{(l-1) !(k-1) !}\right) \frac{x^{r} t^{b-1} q^{w+(n+1 / 2)^{2}}}{r !(b-1) !} \\
= & \left(q \frac{d}{d q} \eta(2 \tau)^{3}\right) \frac{\partial^{3}}{\partial L^{3}} \frac{\partial}{\partial t} \Lambda_{X}-6 \eta(2 \tau)^{3} \frac{\partial}{\partial L} \frac{\partial}{\partial Q} \frac{\partial}{\partial t} \Lambda_{X} .
\end{aligned}
$$

Lemma 4.9. Putting $\lambda_{X}(\tau):=\Lambda_{X}(0,0,0,0, \tau)$ we obtain

$\Lambda_{X}=\exp \left(L / f(\tau)-Q\left(G_{2}(2 \tau)+e_{3}(2 \tau) / 2\right) / f(\tau)^{2}+3 x e_{3}(2 \tau) / f(\tau)^{2}+\theta(\tau)^{-1} t\right) \lambda_{X}(\tau)$.

Proof. Using Remark 3.2 we can reformulate $(0)_{d}-(3)_{d}$ as

$$
\begin{aligned}
\frac{\partial}{\partial t} \Lambda_{X} & =\Lambda_{X} / \theta(\tau), \quad \frac{\partial}{\partial L} \Lambda_{X}=\Lambda_{X} / f(\tau), \\
\frac{\partial}{\partial Q} \Lambda_{X} & =\frac{1}{2} q\left(d \log _{q}(\theta(\tau))\right) \Lambda_{X} / f(\tau)^{2}, \\
\frac{\partial}{\partial x} \Lambda_{X} & =q\left(d \log _{q}\left(\eta(2 \tau)^{3}\right)\right) \Lambda_{X} / f(\tau)^{2}-3 q\left(d \log _{q}(\theta(\tau))\right) \Lambda_{X} / f(\tau)^{2} .
\end{aligned}
$$

So the result follows by Remark 3.2.

To finish the proof of Theorem 3.3 we now only have to identify $\lambda_{X}$.

\section{Lemma 4.10.}

$$
\lambda_{X}=\frac{\Delta(2 \tau)}{f(\tau)^{11}} \exp (\theta(\tau) \sigma(X))=\frac{\Delta(2 \tau)^{2}}{\Delta(\tau) \Delta(4 \tau)} f(\tau) \exp \left(\theta(\tau)^{-1} \sigma(X)\right) .
$$

Proof. We first show that it is enough to prove this result in case $X=\mathbb{P}_{1} \times \mathbb{P}_{1}$. We note that by Lemma 4.9 the statements for a variety $Y$ and $\widehat{Y}=Y \# \overline{\mathbb{P}}_{2}$ are equivalent. It is therefore enough to show it for $\widehat{X}$. $\widehat{X}$ has odd intersection form and $a:=b_{2}(\widehat{X})-1 \geq 2$. So it is homotopy-equivalent to $\mathbb{P}_{2} \# a \overline{\mathbb{P}}_{2}=\left(\mathbb{P}_{1} \times \mathbb{P}_{1}\right) \#(a-1) \overline{\mathbb{P}}_{2}$. As $\delta_{\xi}^{X}$ only depends on the homotopy type of $X$, it is enough to show the result for $\mathbb{P}_{1} \times \mathbb{P}_{1}$. This is in fact the only time in our argument where we use that $\delta_{\xi}^{X}$ depends on the homotopy type of $X$, rather than on $X$ itself.

Let $F, G \in H^{2}\left(\mathbb{P}_{1} \times \mathbb{P}_{1}, \mathbb{Z}\right)$ be the classes of the fibres of the two projections to $\mathbb{P}_{1}$. Let $k \in \mathbb{Z}_{>0}$ and $N:=4 k-1$. Then Lemma 2.9 gives that $\Phi_{F+G, N}^{\mathbb{P}_{1} \times \mathbb{P}_{1}, F+\epsilon G}=$ 
$\Phi_{F+G, N}^{\mathbb{P}_{1} \times \mathbb{P}_{1}, G+\epsilon F}=0$ for all sufficiently small $\epsilon>0$. In particular we have for all $k>0$

$$
(-1)^{k+1} \sum_{\xi \in W_{f+g}^{\mathbb{P}_{1} \times \mathbb{P}_{1}}(F, G)}(-1)^{\varepsilon(F+G, \xi, 4 k-1)} \delta_{\xi}^{\mathbb{P}_{1} \times \mathbb{P}_{1}}\left(2 \check{G}^{4 k-1}\right)=0 .
$$

Here $W_{f+g}^{\mathbb{P}_{1} \times \mathbb{P}_{1}}(F, G)=\left\{(2 n-1) F-(2 m-1) G \mid n, m \in \mathbb{Z}_{>0}\right\}$, and

$$
(-1)^{k+1+\varepsilon(F+G,(2 n-1) F-(2 m-1) G, 4 k-1)}=(-1)^{n+m} .
$$

Applying Lemma 4.9 we get

$$
\operatorname{Coeff}_{q^{0}}\left(\sum_{n, m \in \mathbb{Z}_{>0}}(-1)^{n+m} q^{(2 n-1)(2 m-1) / 2}(2 n-1)^{4 k-1} \frac{\lambda_{\mathbb{P}_{1} \times \mathbb{P}_{1}}(\tau)}{f(\tau)^{4 k-1}}\right)=0 .
$$

Note that, by Definition 4.6, $\lambda_{\mathbb{P}_{1} \times \mathbb{P}_{1}}=q^{-3 / 4} \bar{\lambda}$, where $\bar{\lambda}=\sum l_{i} q^{i}$ is a power series in $q$. Also $f(\tau)=q^{1 / 4} \bar{f}$ with $\bar{f}$ a power series in $q$ with constant term 1 . It is well known $([\mathrm{Ko}],[\mathrm{K}-\mathrm{M}])$ that $\delta_{F-3 G}^{\mathbb{P}_{1} \times \mathbb{P}_{1}}\left((2 \check{G})^{3}\right)=1$. Thus we get $l_{0}=1$. Putting $\lambda_{k}:=\sum_{j<k} l_{j} q^{j},(*)$ gives for each $k \geq 1$ the recursive relation

$$
l_{k}=-\sum_{n, m>0}(-1)^{n+m}(2 n-1)^{4 k-1} \operatorname{Coeff}_{q^{k-2 n m+n+m}}\left(\bar{\lambda}_{k} / \bar{f}^{4 k-1}\right) .
$$

So we see that $\lambda_{\mathbb{P}_{1} \times \mathbb{P}_{1}}$ is uniquely determined by $(*)$. We put

$$
H_{k}(\tau):=\sum_{n, m \in \mathbb{Z}_{>0}}(-1)^{n+m} q^{\frac{1}{4}(2 n-1)(2 m-1)}(2 n-1)^{4 k-1} \Delta(\tau) / f(\tau / 2)^{4 k+10} .
$$

Then the lemma follows from the following lemma (the proof of which is due to Don Zagier).

Lemma 4.11. $\operatorname{res}_{q=0} H_{k}(\tau) \frac{d q}{q}=0$.

Proof. We start by rewriting $H_{k}(\tau)$.

$$
\begin{array}{r}
\sum_{n, m>0}(-1)^{n+m} q^{(n-1 / 2)(m-1 / 2)}(2 n-1)^{4 k-1}=\sum_{d \text { odd }}^{\infty}(-1)^{(d-1) / 2} \sigma_{4 k-1}(d) q^{d / 4} \\
=\frac{1}{2 i}\left(G_{4 k}((\tau+1) / 4)-G_{4 k}((\tau-1) / 4)\right)=: \widetilde{G}_{4 k}(\tau),
\end{array}
$$

where $G_{4 k}(\tau)$ is the Eisenstein series. We write

$$
\phi:=f(\tau / 2)^{2}=(\eta(\tau / 2) \eta(2 \tau) / \eta(\tau))^{4} .
$$

Then we have $H_{k}(\tau)=\widetilde{G}_{4 k}(\tau) \Delta(\tau) / \phi^{2 k+5}$. We want to show that $H_{k}(\tau)$ is a modular form of weight 2 for the $\theta$-group

$$
\Gamma_{\theta}:=\left\{A \in S L(2, \mathbb{Z}) \mid A \equiv\left(\begin{array}{ll}
1 & 0 \\
0 & 1
\end{array}\right) \text { or } A \equiv\left(\begin{array}{ll}
0 & 1 \\
1 & 0
\end{array}\right) \text { modulo } 2\right\} .
$$

The operation of $\Gamma_{\theta}$ is generated by $\tau \mapsto \tau+2$ and $\tau \mapsto-1 / \tau$. We see that $\widetilde{G}_{4 k}(\tau+2)=-\widetilde{G}_{4 k}(\tau)$. Now we write

$$
(-1 / \tau+1) / 4=\frac{(\tau-1) / 4}{4(\tau-1) / 4+1}, \quad(-1 / \tau-1) / 4=\frac{(\tau+1) / 4}{-4(\tau+1) / 4+1},
$$

and use that $G_{4 k}(\tau)$ is a modular form of weight $4 k$ for $S L(2, \mathbb{Z})$, to obtain that

$$
\widetilde{G}_{4 k}(-1 / \tau)=\frac{1}{2 i}\left(\tau^{4} G_{4 k}((\tau-1) / 4)-\tau^{4} G_{4 k}((\tau+1) / 4)\right)=-\tau^{4} \widetilde{G}_{4 k}(\tau) .
$$


Furthermore we see by $\phi(\tau)^{6}=\Delta(\tau / 2) \Delta(2 \tau) / \Delta(\tau)$ that $\phi(-1 / \tau)^{6}=\tau^{12} \phi(\tau)^{6}$, i.e. $\phi(-1 / \tau)=\omega \tau^{2} \phi(\tau)$ for a 6 -th root of unity $\omega$. Putting $\tau:=i$ we get $\phi(i)=$ $-\omega \phi(-1 / i)$, i.e. $\omega=-1$. We also obviously have $\phi(\tau+2)=-\phi(\tau)$. Putting this together and using the fact that $\Delta(\tau)$ is a modular form of weight 12 for $S L(2, \mathbb{Z})$ we finally see that $H_{k}(\tau)$ is a modular form of weight 2 for $\Gamma_{\theta}$. In other words $H_{k}(\tau) d q / q=2 \pi i H_{k}(\tau) d \tau$ is a $\Gamma_{\theta}$-invariant differential form on $\mathbb{H}$, holomorphic out of the cusps $\tau=1$ and $\tau=\infty$ (i.e. $q=0$ ). We show that $H_{k}(\tau)$ is holomorphic at $\tau=1 . \Delta(\tau)$ and $\widetilde{G}_{4 k}(\tau)$ are obviously holomorphic at $\tau=1$. We now put $\tau:=1-1 / z$ and use again that $\Delta(\tau)$ is a modular form of weight 12 for $S L(2, \mathbb{Z})$ and write $1 / 2-1 /(2 z)=\frac{(z-1) / 2}{2(z-1) / 2+1}$ to obtain

$$
\begin{aligned}
\phi(\tau)^{6} & =\frac{\Delta(1 / 2-1 /(2 z)) \Delta(-2 / z)}{\Delta(-1 / z)}=\frac{z^{12} \Delta((z-1) / 2)(z / 2)^{12} \Delta(z / 2)}{z^{12} \Delta(z)} \\
& =-(z / 2)^{12} \frac{\Delta(z)^{2}}{\Delta(2 z)} .
\end{aligned}
$$

So for $z=\infty$, (i.e. $\tau=1$ ) the modular form $\phi(\tau)$ is holomorphic and does not vanish. Thus also $H_{k}(\tau)$ is holomorphic at $\tau=1$. Thus the residue theorem implies that $\operatorname{res}_{q=0}\left(2 \pi i H_{k}(\tau) d \tau\right)=0$.

Remark 4.12. As noted above, we have used that by Conjecture $1.1 \delta_{\xi, N}^{X}$ depends only on the homotopy type $X$ rather than just on $X$ only in the reduction above to $\mathbb{P}_{1} \times \mathbb{P}_{1}$. In particular, without assuming this, our proof still shows Theorem 3.3 for $X$ a rational surface, and therefore also Theorem 3.5.

\section{Possible generalizations}

It should be possible to prove the blowup formulas and also Conjecture 1.1 for 4-manifolds $X$ with $b_{+}(X)=1$ and $b_{1}(X)=0$ (i.e. dropping the assumption that $X$ is simply connected). If we assume these generalizations, then all our arguments in the proof of Theorem 3.3 work in this more general case except for the reduction to $\mathbb{P}_{1} \times \mathbb{P}_{1}$ at the beginning of the proof of Lemma 4.10. So we get

Corollary 5.1. Assume that the blowup formulas 2.6 and Conjecture 1.1 hold for all 4-manifolds $Y$ with $b_{+}(Y)=1$ and $b_{1}(Y)=0$. Then for all $X$ with $b_{+}(X)=1$ and $b_{1}(X)=0$, all $\xi$ in $H^{2}(X, \mathbb{Z})$ with $\xi^{2}<0$ and all $\alpha \in H_{2}(X, \mathbb{Q})$ we have

$$
\delta_{\xi}^{X}(\exp (\alpha z+p x))=\operatorname{res}_{q=0}\left(g_{\xi}^{X}(\alpha z, x, \tau) \lambda_{[X]}(\tau) \Delta(\tau) \Delta(4 \tau) /\left(f(\tau) \Delta(2 \tau)^{2}\right)\right) d q / q,
$$

where $g_{\xi}^{X}$ is the generating function from Theorem 3.3 and $\lambda_{[X]}(\tau)$ is $q^{-3 / 4}$ multiplied by an unknown power series $\bar{\lambda}_{[X]}(q)$ in $q$, which depends only on the equivalence class $[X]$, where $X$ and $Y$ are equivalent if $X \# k \overline{\mathbb{P}}_{2}$ and $Y \# k \overline{\mathbb{P}}_{2}$ are homotopy equivalent for some $k$.

The results of [E-G1] suggest that the dependence of $\lambda_{[X]}(\tau)$ on $X$ should be very simple.

Conjecture 5.2. $\lambda_{[X]}(q)=n_{2} f(\tau) \Delta(2 \tau)^{2} /(\Delta(\tau) \Delta(4 \tau))$, for $n_{2}$ the number of 2torsion points in $H^{2}(X, \mathbb{Z})$. 


\section{REFERENCES}

[Do] Donaldson, S.K., Polynomial invariants for smooth four-manifolds, Topology 29 (1990), 257-315. MR 92a:57035

[E-G1] Ellingsrud, G. and Göttsche, L., Variation of moduli spaces and Donaldson invariants under change of polarization, J. reine angew. Math. 467 (1995), 1-49. CMP 96:02

[E-G2] Ellingsrud, G. and Göttsche, L., Wall-crossing formulas, Bott residue formula and the Donaldson invariants of rational surfaces, preprint 1995.

[E-LP-S] Ellingsrud, G., Le Potier, J. and Strømme, S. A., Some Donaldson Invariants of $\mathbb{C P}^{2}$, preprint 1995.

[F-M] Friedman, R., Morgan, J., On the diffeomorphism types of certain algebraic surfaces, I, J. Diff. Geom. 27 (1988), 297-369. MR 89d:57046

[F-Q] Friedman, R., Qin, Z., Flips of moduli spaces and transition formulas for Donaldson polynomial invariants of rational surfaces, preprint 1994.

[F-S] Fintushel, R., Stern, R.J., The blowup formula for Donaldson invariants, preprint 1994.

[H-B-J] Hirzebruch, F., Berger, T., Jung, R., Manifolds and Modular forms, Vieweg, Braunschweig-Wiesbaden, 1992. MR 94d:57001

[H-P] Hyun, S., Park, J.S. Holomorphic Yang-Mills Theory and Variation of the Donaldson Invariants, hep-th/9503036.

[Ko] Kotschick, D., SO(3)-invariants for 4-manifolds with $b^{+}=1$, Proc. London Math. Soc. 63 (1991), 426-448. MR 92m:57040

[K-L] Kotschick, D., Lisca, P., Instanton Invariants of $\mathbb{C P}^{2}$ via Topology, Math. Ann. 303 (1995), 345-371. CMP 95:17

[K-M] Kotschick, D., Morgan, J., SO(3)-invariants for 4-manifolds with $b^{+}=1 \mathrm{II}$, J. Diff. Geom. 39 (1994), 433-456. MR 95g:57047

[Kr-M] Kronheimer, P., Mrowka, T., Recurrence relations and asymptotics for four-manifold invariants, Bull. Amer. Math. Soc. 30 (1994), 215-221. MR 94k:57046

[L] Leness, T. Blow-up formulae for $S O(3)$-Donaldson invariants, preprint.

[L-Q] Li, W.P., Qin, Z., Lower-degree Donaldson polynomial invariants of rational surfaces, J. Alg. Geom. 2 (1993), 413-442. MR 94f:14032

[Li] Li, J., Algebraic geometric interpretation of Donaldson's polynomial invariants, J. Diff. Geom. 37 (1993), 417-465. MR 93m:14007

[M-O] Morgan, J., Ozsváth, P., private communication.

[O] Ozsváth, P., Some blowup formulas for SU(2) Donaldson polynomials, J. Diff. Geom. 40 (1994), 411-447. MR 95e:57054

[P-T] Pidstrigach, V.Y. Tyurin, A.N., in preparation.

[Q1] Qin, Z., Equivalence classes of polarizations and moduli spaces of sheaves, J. Diff. Geom. 37 (1993), 397-413. MR 94f:14007

[Q2] Qin, Z., Moduli of stable sheaves on ruled surfaces and their Picard groups, J. reine angew. Math. 433 (1992), 201-219. MR 93k:14018

[R] Rankin, R.A., Modular forms and functions, Cambridge University Press, Cambridge, 1977. MR 58:16518

[S-W] Seiberg, N., Witten, E., Electric-Magnetic Duality, Monopole Condensation, and Confinement in $N=2$ Supersymmetric Yang-Mills Theory, Nucl. Phys. B426 (1994), 19-52. MR 95m:81202a

[S] Stern, R., Computing Donaldson Invariants, Lectures for the Park City Geometry Institute, 1994.

[T] Taubes, C.H., in preparation.

[W1] Witten, E., Monopoles and Four-Manifolds, Math. Res. Lett. 1 (1994), 769-796. CMP 95:05

[W2] Witten, E., On S-Duality in Abelian Gauge Theory, hep-th/9505186.

[K-Z] Kaneko, M., Zagier, D., A generalized Jacobi theta function and quasimodular forms, preprint.

Max-Planck-Institut für Mathematik, Gottfried-Claren-Strasse 26, D-53225 Bonn, Germany

Current address: Dipartimento di Matematica, Via Buonarroti 2, I-56127 Pisa, Italy

E-mail address: goettsch@dm.unipi.it 